\title{
Older patients have increased risk of poor outcomes after low-velocity pedestrian-motor vehicle collisions
}

This article was published in the following Dove Press journal:

Pragmatic and Observational Research

26 April 2017

Number of times this article has been viewed

\author{
Gerard A Baltazar' \\ Parker Bassett ${ }^{1}$ \\ Amy J Pate ${ }^{2}$ \\ Akella Chendrasekhar ${ }^{2}$ \\ 'Division of Trauma, Department of \\ Surgery, St. Barnabas Hospital Health \\ System, Bronx, NY, USA; ${ }^{2}$ Division \\ of Trauma, Department of Surgery, \\ Richmond University Medical Center, \\ Staten Island, NY, USA
}

Background: Motor vehicle collisions (MVCs) are a leading cause of injury in the US. While the probability of collision with a pedestrian (PMVC) has declined in recent years, the probability of a pedestrian fatality has risen. Our objective was to determine whether older age impacts potential outcomes in patients involved in low-velocity PMVCs.

Materials and methods: We performed a retrospective-cohort study of adult patients aged $>14$ years involved in low-velocity pedestrian-MVCs $(<15$ miles per hour $[24.14 \mathrm{~km} / \mathrm{h}])$, presenting to an urban level I trauma center from January to November 2013. Subjects were identified via trauma registry and stratified: ages $15-49$ years and $\geq 50$ years. Electronic medical records were reviewed for demographics, vital signs, and laboratory results on initial presentation, presence or absence of systemic inflammatory response syndrome (SIRS), shock index (SI), injuryseverity score (ISS), length of stay (LOS), and survival to discharge. For statistical analysis, $\chi^{2}$ or Student's $t$-tests were utilized.

Results: Our study included 145 patients ( 77 female) with a mean age of $41.9 \pm 3$ years; 95 patients were aged $15-49$ years (mean $31.9 \pm 2.2$ years), and 50 patients were aged $\geq 50$ years or older (mean 62.44 \pm 2.9 years). Mean ISS was $10.05 \pm 1.95$, mean SI was $0.68 \pm 0.03$, and mean LOS was $3.67 \pm 0.57$ days. A total of 41 patients met SIRS criteria on arrival, and nine patients expired $(6.2 \%)$. Mean ISS $(15.64 \pm 4.42$ vs $7.1 \pm 1.64, P<0.001)$ and mean SI $(0.75 \pm 0.07$ vs $0.65 \pm 0.03, P=0.002$ ) were higher in patients aged $\geq 50$ years. Mean LOS was longer in older patients (5.22 \pm 1.14 vs $2.85 \pm 0.58$ days, $P<0.001)$. Older age was associated with SIRS on arrival $(P=0.023)$ and associated with mortality $(P=0.004)$.

Conclusion: Age $\geq 50$ years is associated with greater severity of injury and poor outcomes for patients involved in low-velocity PMVCs. Increased clinical attention and resource allocation should be directed toward older patients after low-velocity PMVCs.

Keywords: motor vehicle collision, pedestrian, older, trauma

\section{Background}

According to the World Health Organization, motor vehicle collisions (MVCs) are a leading cause of traumatic injury, with 20-50 million events occurring annually, resulting in 1.24 million deaths. ${ }^{1}$ The annual cost incurred by the US as a result of MVCs is approximately $\$ 100$ billion. $^{1}$

While the probability of a pedestrian being struck in the course of an MVC (PMVC) has declined in recent years, the probability of a resulting pedestrian fatality has risen. ${ }^{1}$ Some of the increasingly poor outcomes may be related to the increasing age of the trauma population. ${ }^{2}$ Based on prior studies, older patients are at greater risk of being involved in a PMVC and sustaining more severe injuries. ${ }^{3,4}$ Age also correlates with
Correspondence: Gerard A Baltazar Division of Trauma, Department of Surgery, St. Barnabas Hospital Health System, 4422 Third Avenue, Bronx, New York, NY 10457, US

Tel + I 7189606127

Email gbaltazar@sbhny.org 
injury pattern: older patients are more likely to sustain chest and brain trauma compared to younger patients. ${ }^{4,5}$

The growing amount of evidence for age affecting outcome does not necessarily differentiate between the energy of impact. A recent retrospective study found that across all MVC velocities, older patients had twice the risk of mortality and an increased risk of morbidities. ${ }^{6}$ Another study examining PMVCs in general found that the average risk of death for a 70-year-old pedestrian struck at any velocity was similar to the average risk of death for a 30-year-old pedestrian struck at a velocity of $11.8 \mathrm{mph}(19 \mathrm{~km} / \mathrm{h})$ or faster. ${ }^{7}$ In a National Trauma Data Bank study, the shock index (SI; heart rate divided by systolic blood pressure) has been shown to predict mortality among older trauma patients. ${ }^{8}$ Similarly, the presence of systemic inflammatory response syndrome (SIRS; presence of two or more clinical alterations of heart rate, temperature, white blood-cell count, and respiratory rate) may predict outcomes in trauma patients. ${ }^{9}$ Both scores are easily calculated on admission and combined with age and injury-severity score (ISS; complex of abbreviated injury scores for body regions that correlates with mortality, morbidity, and hospital length of stay [LOS] after trauma) may accurately inform resource allocation for PMVC victims.

Data specifically examining low-velocity PMVCs have focused on the pediatric population. ${ }^{10,11}$ As an urban hospital, our population generally ambulates or uses public transport, and low MV velocity is the legally enforced norm. Our aim was to determine whether the age of adult patients who suffered PMVCs at velocities reported to be $15 \mathrm{mph}$ or less impacted outcomes.

\section{Materials and methods}

After receiving a Richmond University Medical Center (RUMC) institutional review board waiver allowing deidentified retrospective data collection without patient written informed consent (RUMC IRB policy 2.11), we retrospectively analyzed the electronic medical records of all adult patients $>14$ years old involved in an emergency medical services (EMS)-reported low-velocity (<15 mph) PMVC who were admitted to an urban level 1 trauma center from January 2013 to November 2013. The RUMC trauma registry was used to identify the study population.

Exclusion criteria were an incomplete data set or mortality within 24 hours of admission (mortality within 24 hours generally denotes no expectation of survival; inclusion of such patients may skew analyses). Data abstracted included demographics, vital signs, evidence of SIRS, ISS, SI, initial platelet level, initial hemoglobin level, initial bicarbonate level, LOS, and survival to discharge.

Subjects were stratified into two groups - those aged 15-49 years and those aged $\geq 50$ years - and then compared (Figure 1). Variances were checked to guide appropriate analysis, and

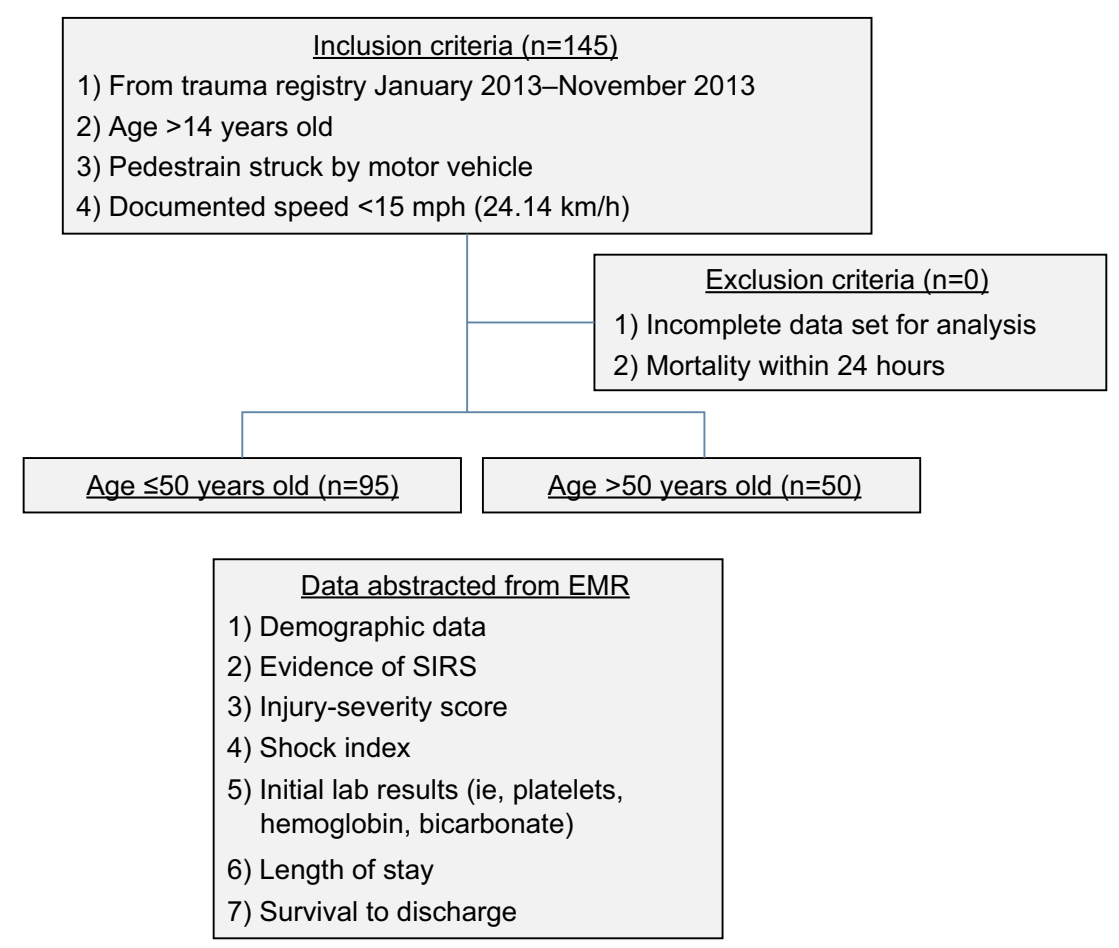

Figure I Retrospective study design of consecutive pedestrians struck by vehicles at low velocity from January I to November 30,20 I 3 . Notes: No patients met exclusion criteria; all patients were included.

Abbreviations: EMR, electronic medical record; SIRS, systemic inflammatory response syndrome. 
statistical comparisons were performed using $\chi^{2}$ analysis or Student's $t$-test as appropriate with Microsoft Excel. Post hoc power analyses were performed using free software available at www. clincalc.com (ClinCalc LLC, Arlington Heights, IL, USA).

\section{Results}

A total of 145 patients were included in the study (68 male and 77 female), with an overall mean age of $41.9 \pm 3$ years; 95 patients (65.5\%) were aged $15-49$ years (mean $31.9 \pm 2.2$ years), and 50 patients $(34.5 \%)$ were aged 50 years or older (mean $62.44 \pm 2.9$ years). There were no exclusions during the study period.

Overall, mean ISS was $10.05 \pm 1.95$, mean SI 0.68 \pm 0.03 , and mean LOS $3.67 \pm 0.57$ days; 41 patients met SIRS criteria on arrival, and nine patients expired (6.2\% mortality). On comparison of younger to older populations, the percentage of females in the cohorts was $51 \%$ and $58 \%$, respectively, although this difference did not achieve statistical significance $(P=0.4)$. However, mean ISS $(15.64 \pm 4.42$ vs $7.1 \pm 1.64$, $P<0.001)$ and mean SI $(0.75 \pm 0.07$ vs $0.65 \pm 0.03, P=0.002)$ were significantly higher in patients aged $\geq 50$ years. Mean LOS was significantly longer in patients aged $\geq 50$ years (5.22 \pm 1.14 vs $2.85 \pm 0.58$ days, $P<0.001$ ) (Figure 2 ).

In addition to these findings, age $\geq 50$ years was associated with the presence of SIRS on arrival $(P=0.023)$ and mortality $(P=0.004)$. Post hoc power analysis for continuous end-point, two-independent-sample study revealed 1 for ISS, SI, and LOS. Post hoc power analysis for dichotomous end point, two-independent-sample study revealed 0.62 for presence of SIRS and 0.76 for mortality. The $\alpha$-level used for these analyses was $P<0.05$. Sex, mean arterial pressure, mean respiratory rate, mean platelets, mean white blood-cell count, and mean bicarbonate levels were similar between the cohorts.

\section{Discussion}

In this retrospective study from a level 1 urban trauma center, we found that age was a critical factor in the severity and outcomes of injury after a low-velocity PMVC. These results seem consistent with previous studies that examined PMVCs more generally ${ }^{7,12,13}$ and may inform hospital systems whose PMVC population may have a large number of low-velocity events.

Although traditionally, older patients are defined as $\geq 65$ years old, various other ages have been used to define an older at-risk population. ${ }^{14,15}$ A recent survey suggested that practitioners use various age definitions ( $>55$ to $>80$ years, with or without consideration of comorbidities) for "geriatric trauma". ${ }^{16}$ In addition, based on our internal hospital data, our population had a high prevalence of multiple major comorbidities among patients younger than 65 years. To capture most effectively data clinically relevant to our population, we opted to classify "older" patients as $\geq 50$ years. Results revealing this age-group is at risk seem to validate this stratification, and will help inform resource allocation for our local patient population.

Comparing ISS and SI between younger and older cohorts, we found a significant increase in each parameter at $\geq 50$ years old, despite uniformly reported low velocity. These differences can be attributed to native physiology in an older population and possibly diminished ability to react or guard against traumatic forces. .,3,17,18 $^{2}$

Variations in anatomical injuries have also been shown to correlate with age, although height of impact will also affect this anatomical variation. An older population has been noted to have more tibial, pelvic, chest, spinal, and brain trauma after PMVCs compared to the pediatric population, whereas hollow viscus and solid organ injuries are similar in the PMVC-trauma population. ${ }^{3}$ Although we did not abstract data on types of injuries, we speculate that variations in injured anatomy may account for the differences in ISS and possibly SI in the older cohort.

Interestingly, our study had equal representation of males and females in both cohorts. Previous studies have found that
A

25

ISS $(P<0.001)$

20

15

10

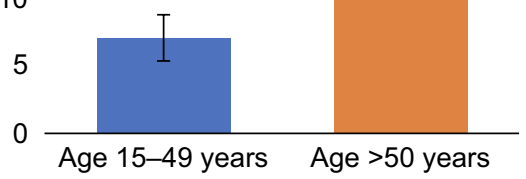

B

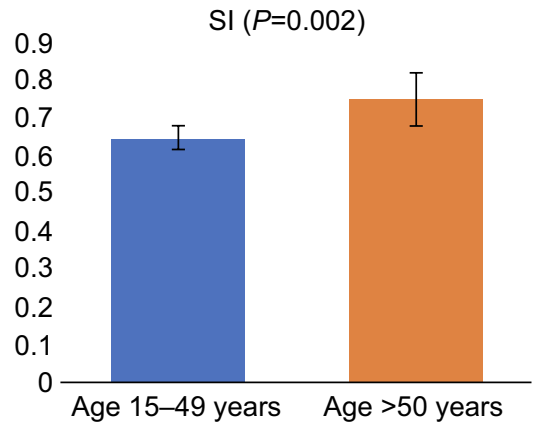

C

$7 \quad \operatorname{LOS}(P<0.001)$

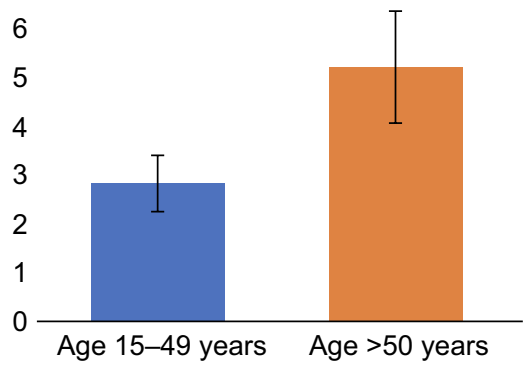

Figure 2 Patients involved in low-velocity pedestrian-motor vehicle collisions.

Notes: Those $\geq 50$ years old (orange) had higher ISS (A), higher SI (B), and longer LOS (C) compared to a younger population (blue). Error bars denote I standard deviation. Abbreviations: ISS, injury-severity score; LOS, length of stay; SI, shock index. 
women are more likely to be involved in PMVCs, and these data are hypothesized to be due to women living longer than men. ${ }^{3,19,20}$ We found no difference in sex distribution between the younger and older cohorts and speculate that because of the high likelihood of pedestrian activity in our urban setting, the risk of PMVCs may be more evenly distributed between sexes.

Patients in the older cohort were also more likely to meet SIRS criteria. Posttraumatic SIRS may exceed that necessary for homeostasis and may contribute to increasing opportunistic infections and complications among all trauma patients, regardless of age or ISS. ${ }^{9,21}$ Studies have noted that older patients may have increased rate and intensity of acute inflammation, ${ }^{22,23}$ and our older cohort may be similarly prone. The older cohort also had a significantly longer hospital LOS. Although the overall mortality rate being low diminished the importance of comparative mortality data, the older cohort also had a significantly lower rate of survival to discharge.

Taken together, our data reflect that older low-velocity PMVC victims have a potential predisposition for poor outcome. We suggest practitioners who care for injured patients pay special attention to older low-velocity PMVC victims and consider directing resource allocation toward the care of these patients.

Our study suffers from the well-known limitations of single-center retrospective-cohort studies, including limited generalizability. Our population size was small but adequately powered (power $>0.8$ ) for ISS, SI, and LOS, although underpowered for SIRS and mortality. Given the urban nature of the study institution's catchment area and the local population's frequent reliance on ambulation, we have a high density of low-velocity PMVCs. We believe our data may reflect similar urban centers. As most data for low-velocity PMVCs are from the pediatric population, despite its limitations, our study adds to the limited data of adult low-velocity PMVCs.

Also, data for velocity of impact were obtained from EMS personnel who quantified the velocity as part of injury descriptors. Such reports may be imprecise compared to final determinations by law enforcement and may limit the validity of our results. However, there seems to be no better way to gauge velocity in an urgent, clinically relevant time frame. In the authors' experience, law enforcement reporting takes considerable time to finalize, often beyond inpatient LOS. Conversely, EMS personnel rapidly provide velocity data that may readily inform clinical decisions and resource allocation. The low overall mortality rate of our study population seems to affirm the accuracy of EMS-personnel reports.
Finally, we did not abstract patient comorbidities. These data may have helped in more adequately characterizing factors contributing to patient outcome, perhaps by multivariate analysis. However, given our use of standardized measures such as SIRS, SI, and ISS, we believe our conclusions are bolstered adequately by our results.

\section{Conclusion}

Age $\geq 50$ years correlates with greater severity of injury and poorer outcomes for patients involved in low-velocity PMVCs. Increased clinical attention and resource allocation should be directed toward older patients after low-velocity PMVCs. Large-scale research is necessary to determine whether these findings are generalizable.

\section{Clinical implications}

Advanced age ( $\geq 50$ years) portends potentially poor outcomes in patients involved in low-velocity PMVCs.

\section{Acknowledgment}

A version of this work was presented at the American College of Chest Physicians Meeting in Austin, TX on October 29, 2014. ${ }^{24}$

\section{Author contributions}

All authors contributed toward data analysis, drafting and revising the paper and agree to be accountable for all aspects of the work.

\section{Disclosure}

The authors report no conflicts of interest in this work.

\section{References}

1. Toroyan T, Peden MM, Laych K. WHO launches second global status report on road safety. Inj Prev. 2013;19(2):150.

2. Chehade M, Gill TK, Renuka V. Low energy trauma in older persons: where to next? Open Orthop J. 2015;9:361-366.

3. Brand $\mathrm{S}$, Otte $\mathrm{D}$, Mueller $\mathrm{CW}$, et al. Injury patterns of seniors in traffic accidents: a technical and medical analysis. World J Orthop. 2012;3(9): 151-155.

4. Demetriades D, Murray J, Martin M, et al. Pedestrians injured by automobiles: relationship of age to injury type and severity. $\mathrm{J} \mathrm{Am} \mathrm{Coll}$ Surg. 2005;199(3):382-387.

5. Brainard BJ, Slauterbeck J, Benjamin JB, Hagaman RM, Higie S. Injury profiles in pedestrian motor vehicle trauma. Ann Emerg Med. 1989;18(8): 881-883.

6. Malone DL, Dunne J, Tracy JK, Putnam AT, Scalea TM, Napolitano LM. Blood transfusion, independent of shock severity, is associated with worse outcome in trauma. J Trauma. 2003;54(5):898-907.

7. Tefft BC. Impact speed and a pedestrian's risk of severe injury or death. Accid Anal Prev. 2013;50:871-878.

8. Pandit V, Rhee P, Hashmi A, et al. Shock index predicts mortality in geriatric trauma patients: an analysis of the National Trauma Data Bank. J Trauma Acute Care Surg. 2014;76(4):1111-1115.

9. Baek JH, Kim MS, Lee JC, Lee JH. Systemic inflammation response syndrome score predicts the mortality in multiple trauma patients. Korean J Thorac Cardiovasc Surg. 2014;47(6):523-528. 
10. Anthikkat AP, Page A, Barker R. Risk factors associated with injury and mortality from paediatric low speed vehicle incidents: a systematic review. Int J Pediatr. 2013;2013:841360.

11. Griffin BR, Watt K, Shields LE, Kimble RM. Characteristics of lowspeed vehicle run-over events in children: an 11-year review. Inj Prev. 2014;20(5):302-309.

12. McElroy LM, Jeurn JJ, Bertleson A, Xiang Q, Szabo A, Weigelt J. A single urban center experience with adult pedestrians struck by motor vehicles. WMJ. 2013;112(3):117-123.

13. Slaughter DR, Williams N, Wall SP, et al. A community traffic safety analysis of pedestrian and bicyclist injuries based on the catchment area of a trauma center. J Trauma Acute Care Surg. 2014;76(4):1103-1110.

14. Kodadek LM, Selvarajah S, Velopulos CG, Haut ER, Haider AH. Undertriage of older trauma patients: is this a national phenomenon? J Surg Res. 2015;199(1):220-229.

15. Goodmanson NW, Rosengart MR, Barnato AE, Sperry JL, Peitzman AB, Marshall GT. Defining geriatric trauma: when does age make a difference? Surgery. 2012;152(4):668-675.

16. Kozar RA, Arbabi S, Stein DM, et al. Injury in the aged: geriatric trauma care at the crossroads. JTrauma Acute Care Surg. 2015;78(6):1197-1209.
17. Baker SP, O’Neill B. The injury severity score: an update. J Trauma. 1976;16(11):882-885.

18. Cannon CM, Braxton CC, Kling-Smith M, Mahnken JD, Carlton E, Moncure M. Utility of the shock index in predicting mortality in traumatically injured patients. J Trauma. 2009;67(6):1426-1430.

19. Bone RC, Balk RA, Cerra FB, et al. Definitions for sepsis and organ failure and guidelines for the use of innovative therapies in sepsis. Chest. 1992;101(6):1644-1655.

20. Reith G, Lefering R, Wafaisade A, et al. Injury pattern, outcome and characteristics of severely injured pedestrian. Scand J Trauma Resusc Emerg Med. 2015;23:56.

21. Stoecklein VM, Osuka A, Lederer JA. Trauma equals danger: damage control by the immune system. J Leukoc Biol. 2012;92(3):539-551.

22. Koo EG, Lai LM, Choi GY, Chan MT. Systemic inflammation in the elderly. Best Pract Res Clin Anaesthesiol. 2011;25(3):413-425.

23. Lineberry C, Stein DE. Infection, sepsis, and immune function in the older adult receiving critical care. Crit Care Nurs Clin North Am. 2014;26(1):47-60.

24. Pate A, Ponukumati S, Chendrasekhar A. Outcomes after low-velocity pedestrian-struck injuries. Chest. 2014;146(4):529A.
Pragmatic and Observational Research

\section{Publish your work in this journal}

Pragmatic and Observational Research is an international, peer-reviewed open access journal that publishes data from studies designed to reflect more closely medical interventions in real-world clinical practice compared with classical randomized controlled trials (RCTs). The manuscript management system is completely online and includes a very quick and fair peer-review

\section{Dovepress}

system. Visit http://www.dovepress.com/testimonials.php to read real quotes from published authors.

Submit your manuscript here: https:/www.dovepress.com/pragmatic-and-observational-research-journal 